\section{Endoscopic ultrasonography of the upper gastrointestinal tract: take a look at the pancreas!}

\section{Filippo Antoninia, Gabriele Capurso ", Giampiero Macarria, Claudio De Angelisc}

Augusto Murri Hospital, Polytechnic University of Marche, Fermo; S. Andrea Hospital, University “La Sapienza”, Rome; Molinette Hospital, Turin, Italy

We read with great interest the article by Martinez et al [1], in which the authors described a study of 298 patients without known pancreatic disease who underwent upper gastrointestinal (GI)-tract endoscopic ultrasound (EUS) to evaluate conditions unrelated to the pancreas. In this study, incidental pancreatic cysts previously undetected by crosssectional imaging studies, such as computed tomography and/or magnetic resonance imaging, were diagnosed in $21.5 \%$ of the patients. Some of these lesions were intraductal papillary mucinous neoplasms (IPMNs) that deserve follow up for the risk of neoplastic evolution. In our opinion, this study raises two important points. First of all, it confirms the high diagnostic accuracy of EUS for evaluating pancreatic abnormalities with a higher resolution than other imaging techniques for small lesions [2,3]. In this regard, we have recently found that, in about $15 \%$ of patients with chronic asymptomatic pancreatic hyperenzymemia, EUS was able to detect pancreatic abnormalities not found with other imaging modalities [3], including IPMN lesions that required follow up for the risk of neoplastic evolution. Likewise, in 57 patients with normal liver enzymes referred for EUS after prior negative imaging studies, abnormal EUS findings were observed in $21 \%$ of patients; they included periampullary diverticula, ampullary adenomas, chronic pancreatitis, biliary stones, and even one pancreatic cancer not previously detected by the other imaging modalities [4].

Furthermore, this study highlights that when EUS examination of the upper GI tract has been planned, a complete exploration of every structure accessible to this techniquenot restricted only to the lesion for which the EUS was indicated-should be mandatory. Unfortunately, this approach is considered time-consuming and, as standard criteria to define the completeness of upper GI-tract EUS examination are lacking, most endoscopists would limit the examination to the initial indication. Indeed, unlike other radiological and endoscopic explorations in which a comprehensive exploration of every visible structure is mandatory, there is no such definition of a standard EUS procedure. Several useful quality indicators for EUS have been published, but a commonly accepted definition of how to perform a complete EUS examination has not yet been provided [5].

Considering that most pancreatic cancer patients are diagnosed as unresectable and that there is no screening program for detection of early pancreatic cancer in the general population, it would probably be worth looking for pancreatic lesions in every upper GI-tract EUS, regardless of the primary indication, especially in patients in otherwise healthy condition.

\section{References}

1. Martínez B, Martínez JF, Aparicio JR. Prevalence of incidental pancreatic cyst on upper endoscopic ultrasound. Ann Gastroenterol 2018;31:90-95.

2. Vila JJ, Jiménez FJ, Irisarri R, et al. Prospective observational study of the incidental findings on endoscopic ultrasonography: should a complete exploration always be performed? Scand J Gastroenterol 2009;44:1139-1145.

3. Antonini F, Belfiori V, Pagano N, et al. Endoscopic ultrasonography may select subjects having asymptomatic chronic pancreatic hyperenzymemia who require a stricter follow-up. Pancreas 2017;46:524-527.

4. Bruno M, Brizzi RF, Mezzabotta L, et al. Unexplained common bile duct dilatation with normal serum liver enzymes: diagnostic yield of endoscopic ultrasound and follow-up of this condition. J Clin Gastroenterol 2014;48:e67-e70.

5. Wani S, Wallace MB, Cohen J, et al. Quality indicators for EUS. Gastrointest Endosc 2015;81:67-80.

${ }^{a}$ Department of Gastroenterology, Augusto Murri Hospital, Polytechnic University of Marche, Fermo (Filippo Antonini, Giampiero Macarri); 'Digestive \& Liver Disease Unit, S. Andrea Hospital, University "La Sapienza”, Rome (Gabriele Capurso); 'Department of Gastroenterology and Hepatology, Molinette Hospital, Turin (Claudio De Angelis), Italy

Conflict of Interest: None

Correspondence to: Filippo Antonini, MD, UOC Gastroenterologia ed Endoscopia Digestiva, Università Politecnica delle Marche, Ospedale “A. Murri”, 63900 Fermo, Italy, e-mail: filippore@yahoo.it

Received 7 March 2018; accepted 7 May 2018; published online 19 June 2018

DOI: https://doi.org/10.20524/aog.2018.0285 